\title{
Long period variable survey in NGC 6822
}

\author{
P. Battinelli ${ }^{1}$ and S. Demers ${ }^{2}$ \\ 1 INAF, Osservatorio Astronomico di Roma Viale del Parco Mellini 84, 00136 Roma, Italy \\ e-mail: battinel@inaf.it \\ 2 Département de Physique, Université de Montréal, CP 6128, Succursale Centre-Ville, Montréal, Qc, H3C 3J7, Canada \\ e-mail: demers@astro.umontreal.ca
}

Received 26 July 2010 / Accepted 4 October 2010

\begin{abstract}
Context. The star-formation history of the intermediate-age population of the Local Group dwarf irregular galaxy NGC 6822 may have been influenced by the often discussed interaction with its HI cloud that might have produced the present polar-ring structure. Aims. We investigate the period distribution of the Mira variables in the central region and the spheroid of NGC 6822.

Methods. We use the new wide-field near-infrared imager CPAPIR to acquire $K_{\mathrm{s}}$ images of a $32^{\prime} \times 32^{\prime}$ area, which includes a good part of the eastern spheroid of NGC 6822.

Results. Periods could be determined for 45 cyclic and semi-regular variables brighter than $\langle K\rangle=17$. The vast majority of the variables have ages between 1 and 3 Gyr.

Conclusions. The period distribution of the long period variables may reflect the proposed interaction with the HI cloud that produces the polar ring.
\end{abstract}

Key words. surveys - stars: AGB and post-AGB - stars: carbon - stars: variables: general - stars: late-type instrumentation: miscellaneous

\section{Introduction}

NGC 6822, a Local Group dwarf irregular galaxy, was the first extragalactic object identified by Hubble (1925), who determined its distance to be 214000 pc. NGC 6822 is quite isolated in the Local Group, $\sim 500 \mathrm{kpc}$ from the Sun and $\sim 700 \mathrm{kpc}$ from M 31. It is located in the constellation Sagittarius toward the Galactic center $\left(\ell=25^{\circ}, b=-18^{\circ}\right)$. Our line of sight to NGC 6822 crosses a relatively heavy stellar foreground making its reddening far from negligible. Today, we adopt for NGC 6822 a distance of $(m-M)_{0}=23.35$, based on the RR Lyrae observations of Clementini et al. (2003), the Cepheids observations of Pietrzyński et al. (2004), and the near-infrared observation of the tip of the red giant branch (TRGB) by Cioni \& Habing (2005). At $470 \mathrm{kpc}$, NGC 6822 is the nearest Magellanic-type galaxy, after the Magellanic Clouds. We adopt a reddening of $E(B-V)=0.24$ as measured by Schlegel et al. (1998). This corresponds to an extinction of $A_{K}=0.08$.

In strong contrast to the description of NGC 6822 given by Barnard (1884) who depicted it as: "a 2' nebula, diffuse and difficult to see", NGC 6822 was later found to be far more extended. Its global structure was first studied by Hodge (1977), who described its bar as a $8^{\prime}$ long structure with a position angle of $10^{\circ}$. In a more detailed investigation by Hodge et al. (1991), the galaxy could be traced to $10^{\prime}$. They adopt the point of view that the galaxy is circular rather than following the shape of the bar. We realize today that the low-density of the periphery of the galaxy can easily be masked by the substantial foreground density seen along the line of sight.

Battinelli et al. (2006) mapped the elliptical spheroid of NGC 6822 to a semi-major axis distance of $36^{\prime}$. From radial velocities of numerous C stars, Demers et al. (2006a) demonstrated that the spheroid is dynamically decoupled from the HI disk (de Blok \& Walter 2000) and rotates at right angles to it.

Hubble's (1925) variable star searches in NGC 6822 detected four variables brighter than the Cepheids, although he could not determine their period and nature. Kayser (1967) repeated Hubble's survey using an impressive number of photographic plates. She found not only Cepheids but some variables with periods over 1000 days. Since then the few published NGC 6822 variable star surveys have targeted Cepheids or RR Lyrae. A number of long period variables (LPV) and semiregular ones (SR) were identified by Antonello et al. (2002). The Araucaria Project also detected a number of LPV in NGC 6822 (Mennickent et al. 2006). In contrast to previous programs, our survey specifically targets the bright AGB stars by observing in the $K_{\mathrm{s}}$ band. We take advantage of a camera with a wide field covering a good part of the spheroid. In Sect. 3.4, we discuss the cross-identification of our variables with those found in previous surveys.

\section{Observations and data reduction}

The observations presented here consist of $K_{\mathrm{S}}$ images obtained in service mode (from April 2005 to March 2007) with the CPAPIR (Caméra Panoramique Proche InfraRouge, see Artigau et al. 2004) imager attached to the CTIO $1.5 \mathrm{~m}$ telescope and operated by the SMARTS consortium. During the 2007 and 2008 observing seasons, observations were acquired with the same camera mounted on the Mont Mégantic $1.6 \mathrm{~m}$ telescope, a telescope with optics similar to the CTIO $1.5 \mathrm{~m}$.

Two overlapping fields were observed, one east of the center and one west of the center that we call $\mathrm{CE}$ and $\mathrm{CW}$, respectively; their coordinates are given in Table 1. This paper presents the results of the CE field, identified in Fig. 1. We currently do not 


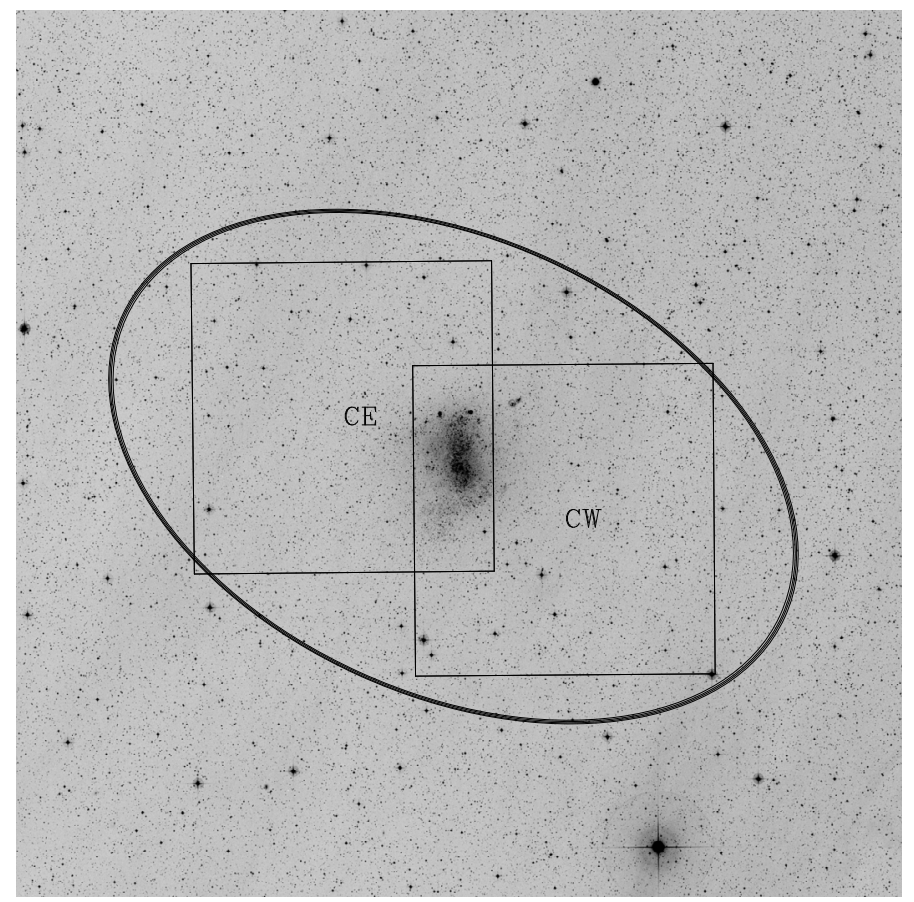

Fig. 1. The outline of the CPAPIR CE and CW fields shown over the central part of NGC 6822. The ellipse defines the stellar spheroid, north at top and east to the left. The field of view is $1.5^{\circ} \times 1.5^{\circ}$.

Table 1. Coordinates of the two CPAPIR fields of NGC 6822.

\begin{tabular}{lc}
\hline \hline Field & RA J2000 Dec \\
\hline CE & $19: 45: 47.0-14: 43: 42$ \\
CW & $19: 44: 13.0-14: 54: 27$ \\
\hline
\end{tabular}

have sufficient observations of the CW field to adequately search for variables.

CPAPIR is based on a $2048 \times 2048$ Hawaii- 2 infrared array detector. With a pixel size of $1.02^{\prime \prime}$, at the $1.5 \mathrm{~m}$ telescope it has a field of view of $34.2^{\prime} \times 34.2^{\prime}$ and $32^{\prime} \times 32^{\prime}$ at the $1.6 \mathrm{~m}$ telescope. One frame consists of five coadded exposures of $5.4 \mathrm{~s}$. The script, for each observation, acquires 100 frames while introducing a 5" dither after each frame. The sky image is built up by evaluating a median of nine frames, centered on the reference frame. This helps to ensure that the temporal variation of the sky transparency is taken into account.

Stars are masked during the sky frame construction. These 100 frames are registered and a median image is built. Our 23 observations correspond to these median images each of a total $2700 \mathrm{~s}$ exposure.

The combined median images are then analysed with DAOPHOT-II and ALLSTAR (Stetson 1994). We select the image obtained in the best seeing as the reference image that is eventually calibrated using 2MASS observations. We first establish the zeropoint magnitude shift between the instrumental magnitudes of the reference image and each of the other images. To do so, we match, using DAOMASTER, individual stars in each image with the stars in the reference image. We then calculate the mean of the magnitude differences selecting only stars with photometric errors $<0.05$. These means are based on between 1100 and 1400 stars. The instrumental magnitudes of the reference image are transformed into the $K_{\mathrm{s}}$ system by crossidentifying our program stars with the 2MASS stars in the fields. We matched 142 2MASS stars in the CE field. The magnitude

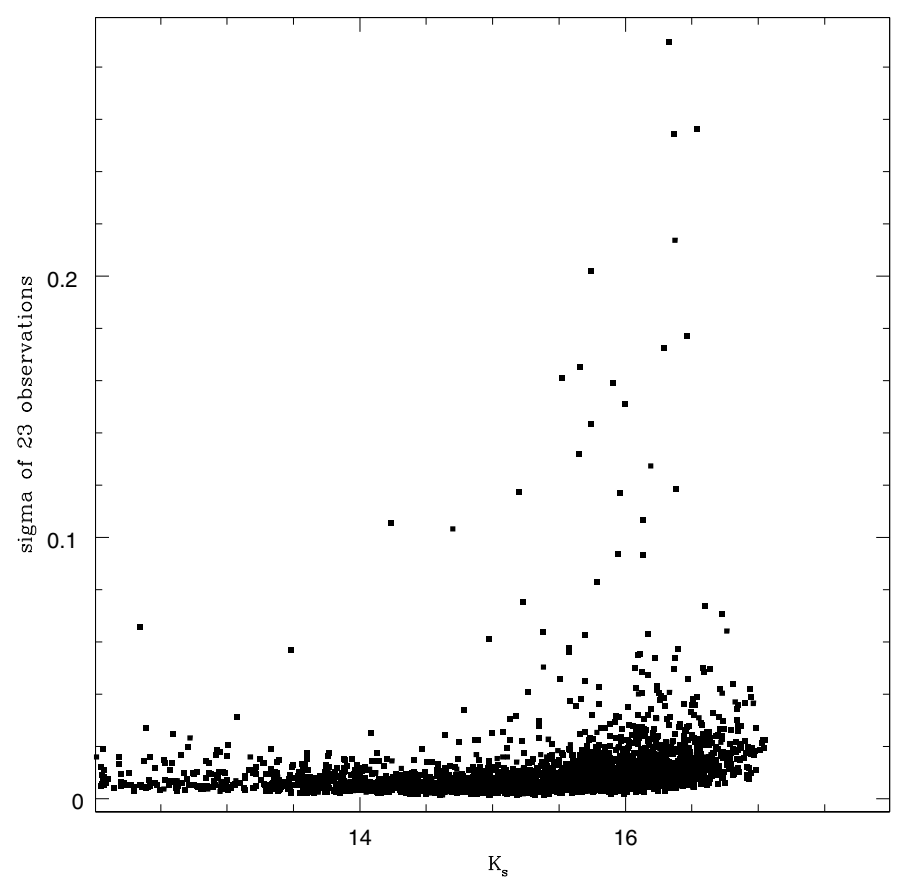

Fig. 2. The standard deviation of 23 observations plotted against the average magnitudes.

shifts of the other images are then applied to all by taking into account the zero point calibration of the reference image.

We can finally produce a table with all the observations with calibrated magnitudes. The dates of the 23 images are listed in Table 2 along with the $F W H M$, in arcsec, of the stars seen in each combined image.

\section{Results}

\subsection{Variable search}

Our first step in the search for variables is to compute the standard deviation in the 23 calibrated magnitudes of each stars, some 4200 stars being matched. We show in Fig. 2 the standard deviation as a function of the apparent magnitudes. Stars one standard deviation above the mean $\sigma$ of the stars of similar magnitudes are considered as candidates for variability. The lower thresholds for $\sigma$ range from 0.015 mag for $K<15$ then rise to reach $0.04 \mathrm{mag}$ at $K=16.5$. To our data we also applied the detection technique developed by Welch \& Stetson (1993). We however modified the method to be used with only one filter band. No additional candidates were detected.

A period search was performed for hundreds of candidates using three approaches: first a simple sine curve fitting; second a phase dispersion minimization code (Stellingwerf 1978); and finally a Fisher randomization test to confirm the reality of the period. Light curves were then plotted and the period producing the tightest fit was adopted. For both methods, the quality of the period determination is expressed by the parameter $\theta$ (Stellingwerf 1978), where $\theta$ is defined as the ratio of the variance in the magnitudes $\left(\sigma^{2}\right)$ and the variance in a subset of the magnitudes $\left(s^{2}\right)$. If the selected period is not the true one, $s^{2} \approx \sigma^{2}$, whereas if the period is correct $\theta \approx 0$.

We list, in Table 3, the J2000 equatorial coordinates of the 33 cyclic variables identified along with their period, the $\theta$, the amplitude, and the mean magnitude. The accuracy of the accepted period is assessed in the following way. For each cyclic 
Table 2. Dates of the CE field observations.

\begin{tabular}{|c|c|c|c|c|c|c|c|}
\hline Name & Date & JD $2450000+$ & $F W H M$ & Name & Date & JD $2450000+$ & $F W H M$ \\
\hline CE001 & $24 / 05 / 2005$ & 3515.826 & 3.8 & CE013 & 01/07/2006 & 3918.679 & 2.3 \\
\hline CEOO2 & $06 / 06 / 2005$ & 3528.717 & 1.8 & CE014 & $19 / 07 / 2006$ & 3936.701 & 2.5 \\
\hline CE003 & $20 / 06 / 2005$ & 3542.637 & 2.4 & CE015 & $23 / 08 / 2006$ & 3971.528 & 2.7 \\
\hline CEOO4 & $06 / 07 / 2005$ & 3558.693 & 1.9 & CE016 & $07 / 09 / 2006$ & 3986.511 & 2.1 \\
\hline CE005 & $01 / 08 / 2005$ & 3584.616 & 1.9 & CE017 & $27 / 10 / 2006$ & 4037.472 & 2.6 \\
\hline CEOO6 & $16 / 08 / 2005$ & 3599.580 & 2.3 & CE018 & $22 / 03 / 2007$ & 4182.865 & 2.2 \\
\hline CE007 & $30 / 09 / 2005$ & 3644.540 & 2.9 & CE019 & $19 / 08 / 2007$ & 4332.575 & 2.5 \\
\hline CE008 & $17 / 10 / 2005$ & 3661.563 & 2.8 & CEO20 & $19 / 09 / 2007$ & 4363.502 & 3.1 \\
\hline CEOO9 & $13 / 04 / 2006$ & 3839.832 & 2.9 & CE021 & $02 / 07 / 2008$ & 4650.634 & 3.3 \\
\hline CE010 & $06 / 05 / 2006$ & 3862.799 & 2.6 & CE022 & $20 / 08 / 2008$ & 4699.539 & 2.9 \\
\hline CE011 & $14 / 06 / 2006$ & 3901.679 & 2.1 & CE023 & $21 / 08 / 2008$ & 4700.613 & 1.8 \\
\hline CE012 & $30 / 06 / 2006$ & 3917.607 & 2.4 & & & & \\
\hline
\end{tabular}

Table 3. Cyclic variables discovered in NGC 6822.

\begin{tabular}{ccccccc}
\hline \hline id & RA \& Dec J2000 & $P($ days $)$ & $\log (P)$ & $\theta$ & Amp. & $\langle K\rangle$ \\
\hline 01 & $19: 44: 47.21-14: 45: 03.78$ & 576 & 2.76 & 0.05 & 1.0 & 15.80 \\
02 & $19: 44: 47.78-14: 51: 41.55$ & 339 & 2.53 & 0.13 & 1.5 & 17.25 \\
03 & $19: 44: 48.98-14: 38: 05.38$ & 326 & 2.51 & 0.09 & 0.7 & 15.67 \\
04 & $19: 44: 51.16-14: 40: 51.21$ & 403 & 2.60 & 0.20 & 0.7 & 15.14 \\
05 & $19: 44: 51.90-14: 49: 54.48$ & 777 & 2.89 & 0.03 & 0.9 & 16.25 \\
06 & $19: 44: 51.98-14: 47: 25.32$ & 437 & 2.64 & 0.07 & 1.2 & 16.32 \\
07 & $19: 44: 57.30-14: 52: 13.04$ & 447 & 2.65 & 0.15 & 1.0 & 16.20 \\
08 & $19: 45: 00.20-14: 46: 04.09$ & 674 & 2.83 & 0.10 & 0.9 & 14.25 \\
09 & $19: 45: 01.30-14: 41: 11.65$ & 619 & 2.79 & 0.32 & 0.4 & 15.80 \\
10 & $19: 45: 01.89-14: 47: 32.27$ & 124 & 2.09 & 0.20 & 0.4 & 14.80 \\
11 & $19: 45: 02.12-14: 53: 16.52$ & 992 & 3.00 & 0.13 & 1.5 & 14.85 \\
12 & $19: 45: 04.04-14: 44: 25.14$ & 436 & 2.64 & 0.26 & 0.9 & 15.75 \\
13 & $19: 45: 04.91-14: 44: 05.17$ & 466 & 2.67 & 0.21 & 0.5 & 15.30 \\
14 & $19: 45: 05.52-14: 45: 05.04$ & 394 & 2.59 & 0.16 & 0.8 & 15.80 \\
15 & $19: 45: 08.20-14: 48: 31.61$ & 633 & 2.80 & 0.10 & 1.1 & 16.25 \\
16 & $19: 45: 08.56-14: 44: 22.26$ & 223 & 2.37 & 0.31 & 0.6 & 16.80 \\
17 & $19: 45: 11.49-14: 44: 48.64$ & 1100 & 3.04 & 0.05 & 1.9 & 16.60 \\
18 & $19: 45: 11.85-14: 33: 18.00$ & 470 & 2.67 & 0.03 & 1.0 & 16.30 \\
19 & $19: 45: 11.90-14: 41: 51.18$ & 448 & 2.65 & 0.06 & 1.3 & 16.25 \\
20 & $19: 45: 12.02-14: 52: 43.53$ & 302 & 2.48 & 0.28 & 0.5 & 15.85 \\
21 & $19: 45: 12.75-14: 47: 32.24$ & 613 & 2.79 & 0.14 & 0.7 & 15.95 \\
22 & $19: 45: 15.69-14: 37: 28.44$ & 456 & 2.66 & 0.13 & 0.7 & 16.02 \\
23 & $19: 45: 18.07-14: 49: 05.59$ & 949 & 2.98 & 0.10 & 0.6 & 15.80 \\
24 & $19: 45: 25.25-14: 43: 11.43$ & 670 & 2.83 & 0.10 & 0.8 & 15.70 \\
25 & $19: 45: 27.63-14: 55: 51.57$ & 257 & 2.41 & 0.10 & 0.7 & 16.05 \\
26 & $19: 45: 32.28-14: 44: 10.21$ & 295 & 2.47 & 0.18 & 0.6 & 16.10 \\
27 & $19: 45: 32.64-14: 51: 17.27$ & 605 & 2.78 & 0.05 & 1.3 & 15.65 \\
28 & $19: 45: 37.13-14: 47: 32.35$ & 336 & 2.53 & 0.34 & 0.5 & 15.82 \\
29 & $19: 45: 42.23-14: 46: 50.88$ & 605 & 2.78 & 0.07 & 1.0 & 15.70 \\
30 & $19: 46: 04.75-14: 41: 40.63$ & 353 & 2.55 & 0.18 & 0.7 & 15.70 \\
31 & $19: 46: 05.44-14: 50: 34.70$ & 1300 & 3.11 & 0.16 & 1.4 & 16.60 \\
32 & $19: 46: 28.94-14: 45: 54.90$ & 872 & 2.94 & 0.14 & 0.8 & 16.30 \\
33 & $19: 46: 44.24-14: 42: 59.33$ & 465 & 2.67 & 0.32 & 0.6 & 16.40 \\
\hline & & & & & &
\end{tabular}

variable we calculate the absolute value of the period difference divided by the mean period determined by the two above methods. For the 33 cyclic variables, we obtain a relative mean error of $5 \pm 9$ percent.

Unfortunately, not all variable star candidates have $23 \mathrm{ob}-$ servations. Some of the last Mont Megantic images indeed have brighter limiting magnitudes thus producing fainter stars with substantial photometric error. For this reason, we also investigated a set of candidates with 19 or more observations.

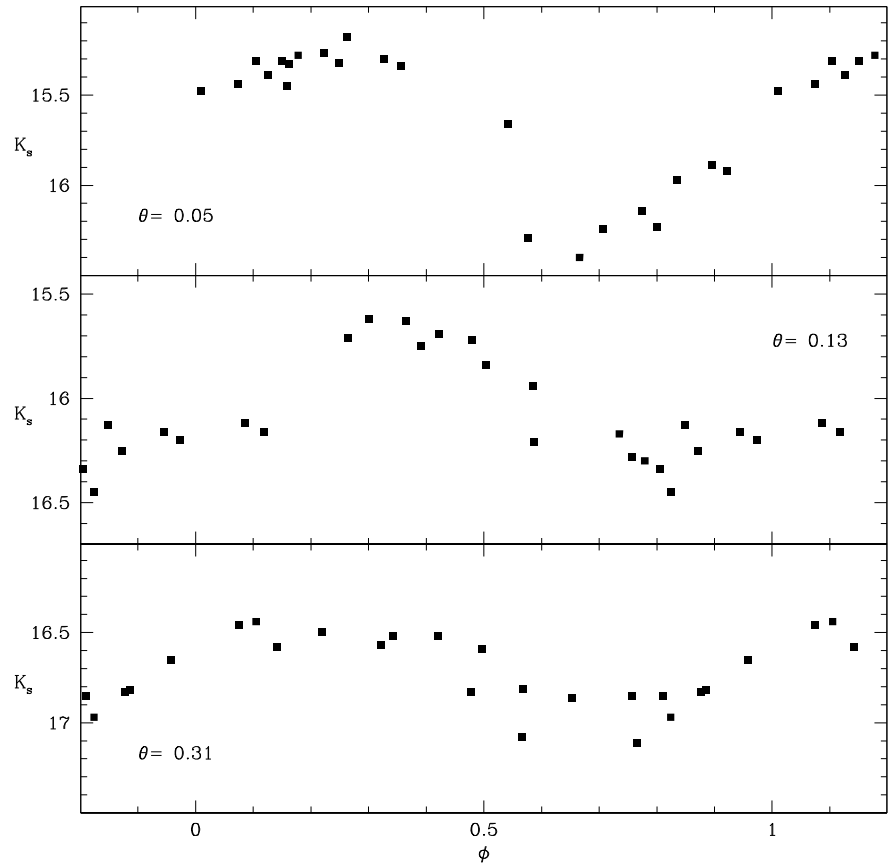

Fig. 3. Light curves of cyclic variables 1,7 and 21 are shown.

Examples of light curves, for three different $\theta$, are presented in Fig. 3.

\subsubsection{Uncertainties in magnitudes and periods}

To provide an idea of the photometric errors involved, we present in Fig. 4 the light curve, with error bars, of two variables of different mean magnitudes. The error bars correspond to the photometric errors determined by DAOPHOT. For the brighter star, we see that errors are of the order of $0.06 \pm 0.02 \mathrm{mag}$, while for the fainter variable errors are slightly larger at $0.08 \pm 0.03$. The uncertainties in the $\langle K\rangle$ listed in Tables 3 and 4 are smaller than $0.1 \mathrm{mag}$. These mean magnitudes are estimated in two ways. First by taking the average of all points and second by hand drawing a curve through the points and calculating the mean of $K_{\max }$ and $K_{\min }$.

\subsection{Semi-regular and irregular variables}

We find 31 variables, which are listed with numbers between 100 and 130, with irregular light variations. For some of them, 
A\&A 525, A69 (2011)

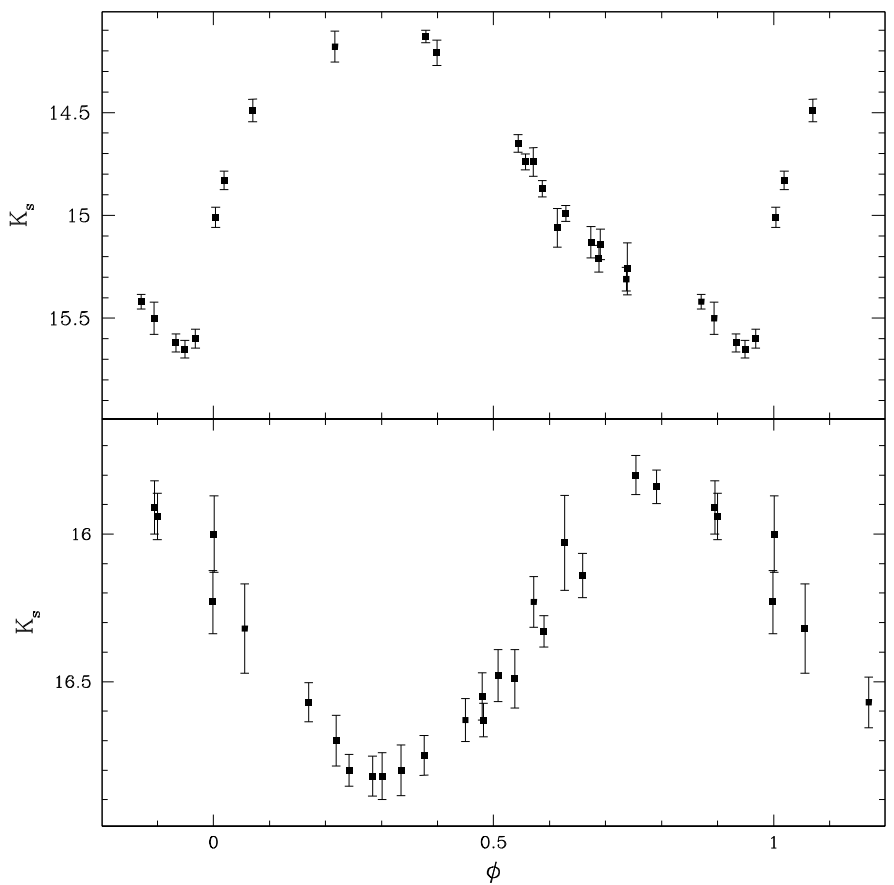

Fig. 4. Light curves of Mira 11 on top and Mira 18 in the lower panel.

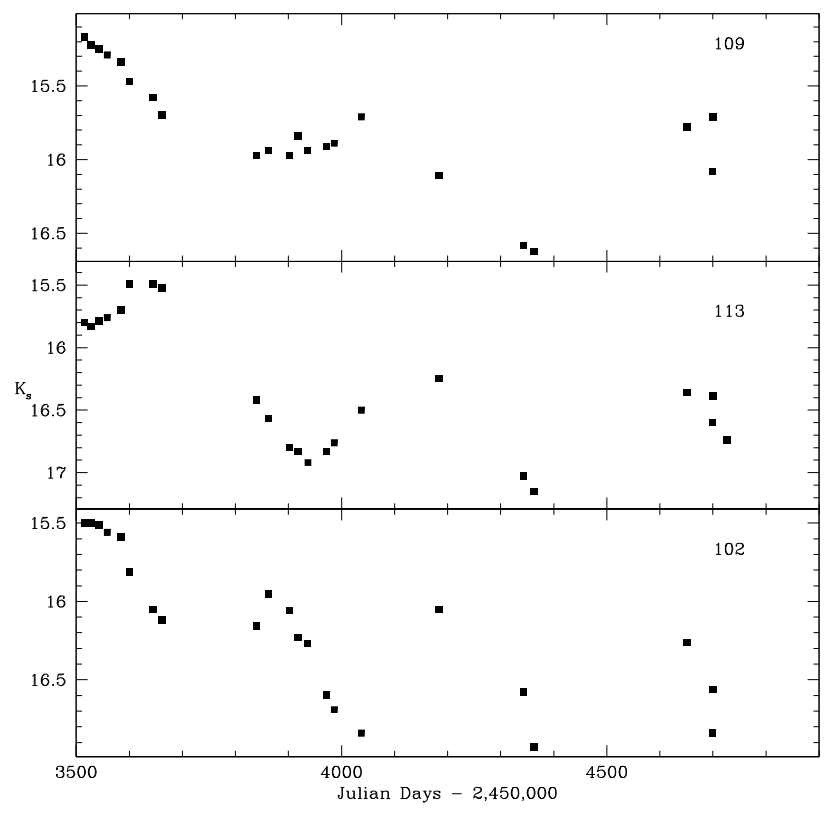

Fig. 5. Examples of three irregular variables, a period of 470 days can be assigned to star 113 .

we can assign a periodicity. These stars are listed in Table 4. We show three examples in Fig. 5.

\subsection{Photometric properties of the variables}

Even though we have obtained only the $K_{\mathrm{s}}$ magnitude of the variables, we can cross-identify our stars with observations of the stellar population of NGC 6822, in various filter bands, available in the literature. Both C-rich and O-rich AGB stars of NGC 6822 were identified with narrow-band filters pioneered by
Table 4. Semi-regular and irregular variables discovered in NGC 6822.

\begin{tabular}{ccccc}
\hline \hline id & RA \& Dec J2000 & $\sim P($ days $)$ & $\log (P)$ & $\langle K\rangle$ \\
\hline 100 & $19: 44: 48.41-14: 53: 58.40$ & & & 16.5 \\
101 & $19: 44: 48.82-14: 47: 21.96$ & & & 16.7 \\
102 & $19: 44: 52.12-14: 46: 35.06$ & & & 16.2 \\
103 & $19: 44: 52.80-14: 45: 29.11$ & & & 15.9 \\
104 & $19: 44: 52.87-14: 43: 42.16$ & & & 17.1 \\
105 & $19: 44: 54.30-14: 43: 31.53$ & & & 15.9 \\
106 & $19: 44: 54.32-14: 46: 02.41$ & 935 & 2.97 & 15.8 \\
107 & $19: 44: 56.27-14: 46: 23.51$ & & & 15.7 \\
108 & $19: 44: 58.19-14: 54: 41.46$ & & & 16.9 \\
109 & $19: 44: 59.86-14: 46: 54.34$ & & & 15.8 \\
110 & $19: 45: 00.08-14: 36: 12.82$ & & & 15.6 \\
111 & $19: 45: 00.39-14: 44: 10.39$ & & & 16.4 \\
112 & $19: 45: 00.82-14: 46: 09.59$ & & & 16.1 \\
113 & $19: 45: 01.77-14: 46: 34.59$ & 470 & 2.67 & 16.3 \\
114 & $19: 45: 01.88-14: 46: 09.29$ & & & 16.4 \\
115 & $19: 45: 02.29-14: 50: 52.36$ & & & 16.4 \\
116 & $19: 45: 04.04-14: 44: 25.14$ & & & 15.9 \\
117 & $19: 45: 05.65-14: 41: 39.87$ & 440 & 2.64 & 16.6 \\
118 & $19: 45: 09.87-14: 41: 51.76$ & 462 & 2.66 & 15.8 \\
119 & $19: 45: 11.79-14: 41: 29.23$ & & & 15.9 \\
120 & $19: 45: 12.42-14: 54: 12.80$ & 352 & 2.55 & 16.1 \\
121 & $19: 45: 18.99-14: 35: 02.29$ & 149 & 2.17 & 16.9 \\
122 & $19: 45: 22.09-14: 45: 44.09$ & & & 16.8 \\
123 & $19: 45: 27.63-14: 44: 17.66$ & 566 & 2.75 & 15.8 \\
124 & $19: 45: 33.03-14: 52: 22.91$ & 389 & 2.59 & 15.7 \\
125 & $19: 45: 47.86-14: 41: 32.38$ & & & 16.2 \\
126 & $19: 45: 57.32-14: 38: 17.33$ & & & 16.4 \\
127 & $19: 45: 59.52-14: 42: 10.16$ & 567 & 2.75 & 15.9 \\
128 & $19: 46: 00.40-14: 36: 57.74$ & 319 & 2.50 & 16.2 \\
129 & $19: 46: 07.87-14: 53: 58.43$ & 245 & 2.39 & 16.1 \\
130 & $19: 46: 34.44-14: 44: 44.52$ & 187 & 2.27 & 16.7 \\
\hline & & & &
\end{tabular}

Cook et al. (1986). The (CN-TiO) colour index indeed allows the distinctiontion of $\mathrm{C}$ and $\mathrm{M}$ AGB stars. Following Letarte et al. (2002), we call $\mathrm{C}$ stars those with a $(R-I)>0.90$ and $(\mathrm{CN}-$ $\mathrm{TiO})>0.3$. The variables cross-identified are listed in Table 5. Near-infrared observations of the central part of NGC 6822 were derived by Cioni \& Habing (2005) and Kang et al. (2006), while the outer parts of the spheroid was observed by Demers et al. (2006b). Here we call C stars those with $(J-K)_{0}>1.4$ (Battinelli et al. 2007). We note that the two C-star criteria are not always compatible (Battinelli \& Demers 2011) (see for example stars 16 and 100); C stars may have $(J-K)_{0}$ bluer than 1.4 . The variability of the stars affects their observed colours. Forty-one of the 64 variables are cross-identified, leading to $31 \mathrm{C}$ stars out of 41. There are several reasons why all the variables could not be identified with the published photometry. Our field extends well outside the other surveys; near the center of NGC 6822, there is certainly a crowding problem, which makes cross-identification difficult. We also cross-identified our variables with the $i^{\prime}, g^{\prime}, r^{\prime}$ database of Battinelli et al. (2006), which extends across a wider area. The differentiation between $\mathrm{C}$-rich and O-rich giants is difficult with these bands. We found no new C-star candidates.

In Fig. 6, we show the positions of the variables on the $I$ versus (vs.) $R-I$ plane. Only variables that can be matched to our database are plotted. Most of them are redder and below the tip of the red giant branch. However, we emphasize that their magnitude and colour are based on only one observation, which may certainly explain the wide spread of magnitudes. In the $K$ vs. $J-K$ plane, our variables would all be above the TRGB. Unfortunately, no such CMD can be produced because we cannot evaluate the $J-K$ colours. 
Table 5. Variables with known colour indices.

\begin{tabular}{rccccccccc}
\hline id & $(\mathrm{CN}-\mathrm{TiO})$ & $(J-K)$ & Ref & Comment & id & $(\mathrm{CN}-\mathrm{TiO})$ & $(J-K)$ & Ref & Comment \\
\hline 3 & -0.049 & & & & & & & & \\
5 & -0.128 & 1.12 & 2 & M star & 104 & 0.404 & & & C star \\
8 & & 1.27 & 3 & M star & 105 & 0.192 & 2.02 & 2 & C star \\
9 & 0.475 & 1.64 & 2 & C star & 106 & 0.285 & 1.82 & 2,3 & C star \\
11 & & 2.38 & 2 & C star & 107 & 0.239 & 2.43 & 3 & C star \\
13 & 0.419 & & & C star & 108 & 0.218 & & & \\
14 & -0.411 & 1.03 & 2 & M star & 109 & & 3.61 & 3 & C star \\
16 & 0.527 & 1.25 & 2 & C star & 110 & -0.108 & & & \\
19 & 0.126 & 2.14 & 2 & C star & 111 & 0.277 & 2.00 & 3 & C star \\
20 & 0.300 & 2.62 & 2 & C star & 112 & -0.078 & 2.84 & 3 & C star \\
23 & 0.452 & 1.75 & 2 & C star & 113 & & 2.96 & 3 & C star \\
25 & 0.607 & & & C star & 114 & 0.454 & 1.90 & 3 & C star \\
26 & 0.369 & & & C star & 115 & 0.493 & 1.88 & 2 & C star \\
28 & 0.134 & & & C star & 118 & & 0.71 & 2 & K star \\
30 & 0.333 & & & C star & 120 & -0.029 & 3.14 & 2 & C star \\
31 & 0.342 & 1.87 & 1 & C star & 121 & -0.488 & & & M star \\
32 & & 2.15 & 1 & C star & 122 & & 2.34 & 2 & C star \\
100 & 0.417 & 1.18 & 2 & C star & 125 & 0.499 & & & C star \\
101 & 0.398 & 1.97 & 2 & C star & 129 & 0.438 & & & C star \\
102 & & 2.21 & 2,3 & C star & 130 & & 0.53 & 1 & \\
103 & 0.436 & 1.69 & 3 & C sta & \\
\hline
\end{tabular}

References. Near infrared references: (1) Demers et al. (2006b); (2) Cioni \& Habing (2005); (3) Kang et al. (2006).

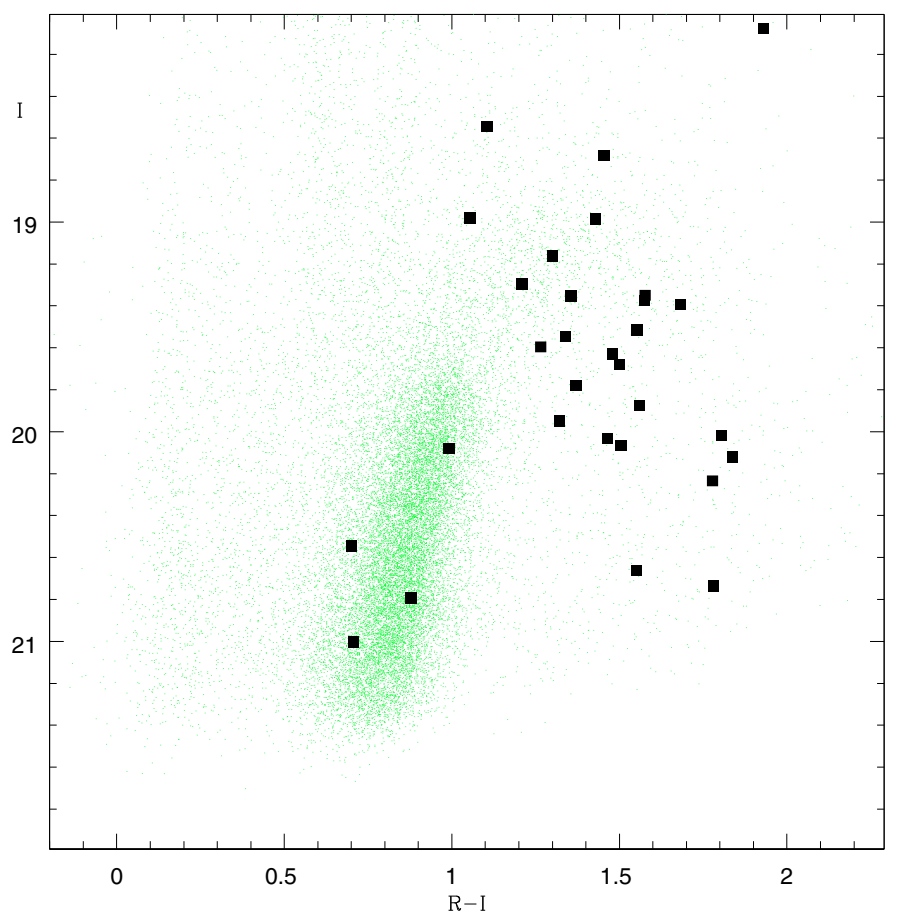

Fig. 6. The variables (big squares) with optical magnitudes and colours are displayed on the CMD of the central region of NGC 6822 (small green dots).

\subsection{Other variable star surveys}

In NGC 6822, Kayser (1967) identified 11 long-period variables with periods between 200 and 2500 days. Unfortunately, coordinates of only six of them are available in SIMBAD and they do not correspond to any of our variables. They match stars that are constant in light, suggesting that the coordinates are not accurate. Antonello et al. (2002) published a list of several dozens long-period and irregular variables in a small $3.8^{\prime} \times 3.8^{\prime}$ field in
NGC 6822. There are only three of our variables in their small field and we find no match. As part of the Araucaria project, Mennickent et al. (2006) identified a number of bright variables in NGC 6822 . Their $35^{\prime} \times 35^{\prime}$ field, presumably centered on the galaxy, should partly overlap with ours. In contrast to the previously described photometric cross-identification, we find the Araucaria variables difficult to match to our database: we find differences smaller than $\sim 1.5^{\prime \prime}$ for the very few matches obtained. They are at the limit of our acceptance criterion. We suspect that the two sets of coordinates are not compatible. The only periodic variable matched is our v16 with their v90. They quote a period of 170 days, while we find 223 days. Among their "non-periodic" stars we have five matches, three of our cyclic variables and two irregular ones. We could relax the acceptance criterion to $2^{\prime \prime}$ but we worry about the spurious matches, especially in the central crowded region. The large number of variables identified by Mennickent et al. (2006) contrasts with our few dozen. To explain this difference, we cross-identified our full database with their variables. We found dozens of matches (within 1.5"). They, however, correspond to faint stars for which we do not have a sufficient number of observations for a period search. These stars were not retained for our period search.

\section{Discussion}

In Fig. 7, we display the $\langle K\rangle \log P$ diagram of the periodic and semi-periodic variables. The solid line traces the $P-L$ relation of C-rich Miras in the LMC (Whitelock et al. 2008), adjusted for the distance and reddening of NGC 6822 by adding 23.43 to the absolute magnitudes. The metallicity effect on the magnitude of Miras was discussed by Ita et al. (2004), who estimate that the SMC Miras are brighter than the LMC Miras of the same period by $\approx 0.13 \mathrm{mag}$. Since the abundance of NGC 6822 is inbetween that of the two Magellanic Clouds, we should expect a shift of smaller than 0.1 mag. The TRGB of NGC 6822 is at $K \approx 17.2$ (Kang et al. 2006), this means that all the stars displayed in Fig. 5 are AGB stars located above the tip. This 


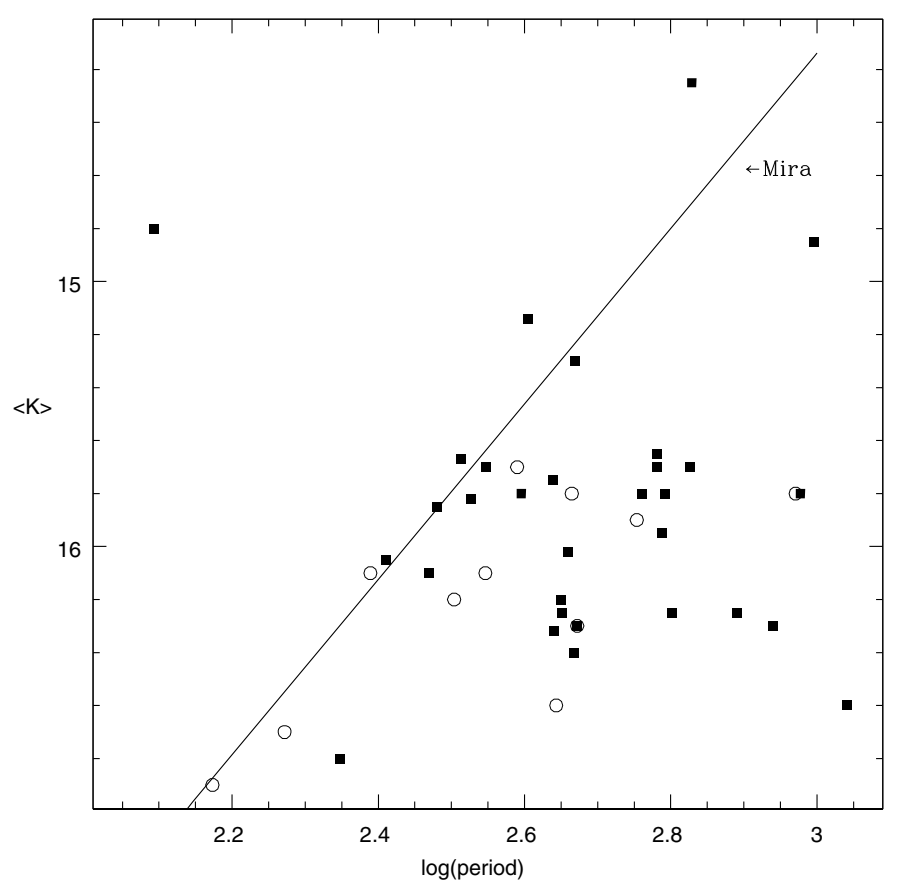

Fig. 7. The mean $K$ magnitude of the variables exhibits only a weak correlation with their periods. Open circles represent the semi-regular variables. The line represents the $P-L$ relation of LMC Miras shifted to the modulus of NGC 6822 .

figure shows that Miras with periods shorter than $\sim 150$ days are fainter than $\langle K\rangle \sim 17$ and therefore not observed by the present survey. The obvious large scatter is expected for LPVs selected from NIR observations. The dusty variables, which are usually not detected by visual observations, have a fainter $K$ magnitude than those without dust.

\subsection{Age distribution}

Hodge (1980) found evidence that a relatively large amount of star formation occurred in the central region of NGC 6822, between 75 and 100 Myr ago. Gallart et al. (1996) showed that the star formation increased by a factor of 2-6 between 100 and 200 Myr ago. de Blok \& Walter (2000) also suggested that an $\mathrm{HI}$ interaction some $100 \mathrm{Myr}$ ago gave birth to the current star formation. The Miras discovered in the present study are representative of substantially older populations.

It has been well-established that the period of a Mira represents a good indicator of the stellar population to which it belongs. Longer-period Miras are expected to have higher mass progenitors, thus belong to a younger population (Iben $\&$ Renzini 1983). Therefore, the period of a Mira is related to its age (Habing \& Whitelock 2004). For example, Feast et al. (2006) determined, from the velocity dispersion of Galactic Miras, that those with periods longer than 500 days are $\approx 1$ Gyr old, while those with $P \approx 375$ days have ages of $\sim 3$ Gyr. Following Habing $\&$ Whitelock (2004), we adopt the following period/age groups: variables with $P<250$ days are understood to be older than $\sim 5$ Gyr, those with periods between 250 and 550 days are of intermediate age between 1 and $3 \mathrm{Gyr}$, while those with longer periods are 1 Gyr or younger.

The period distribution of the Miras and SR variables of NGC 6822 is presented in the first panel of Fig. 8. There are many more variables that have ages between 1 and 3 Gyr than variables younger than 1 Gyr. This implies that there is an

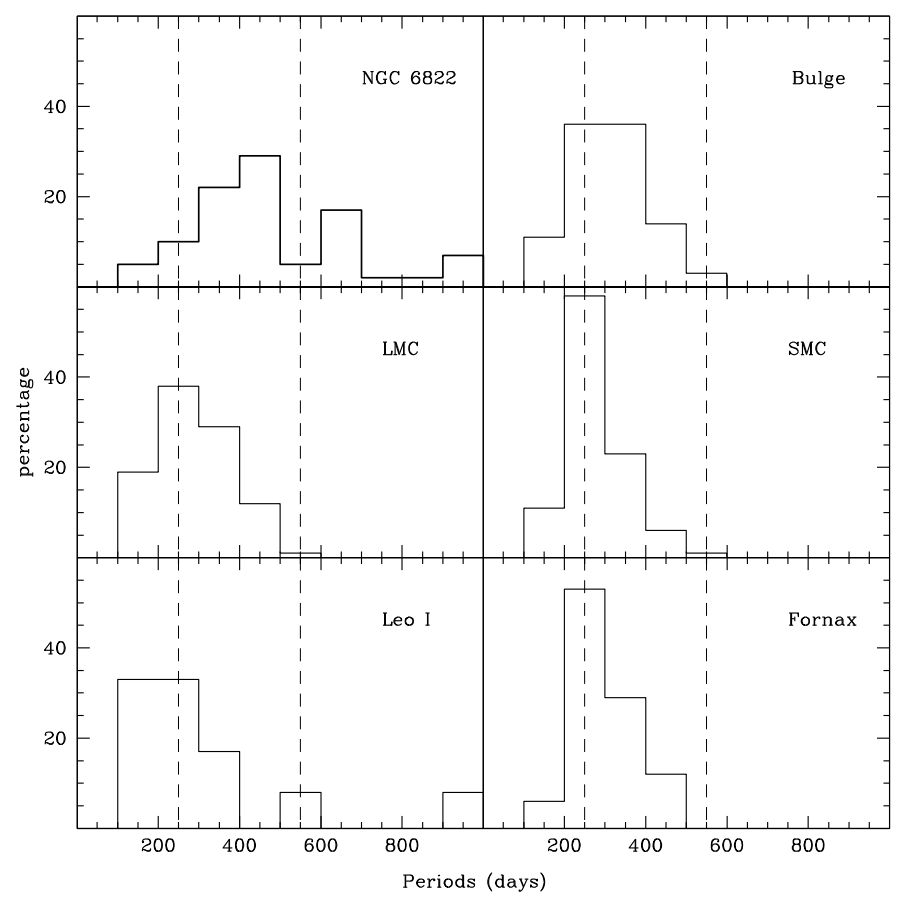

Fig. 8. Period distribution of the LPVs in various galaxies. The dashed lines separate the three age groups, from left to right: $>5 \mathrm{Gyr}, 1$ to $3 \mathrm{Gyr}$ and $<1$ Gyr.

increase in the star-formation rate more than 1 Gyr ago. A search of the literature allows us to compare the period distributions of a few populations of Miras. Searches for Miras have not yet been performed for a good number of Local Group galaxies. However, a few surveys are in progress (e.g. Lorenz et al. 2011).

The period distribution of the Galactic bulge Miras, observed by the OGLE-II survey, was compiled by Groenewegen \& Blommaert (2006), while those of the Magellanic Clouds, also from the OGLE-II survey, was published by Groenewegen (2004). Data for the dwarf spheroidal galaxies are from Menzies et al. (2010) for Leo I and Whitelock et al. (2009) for Fornax.

The period distribution of NGC 6822 contrasts with those of other galaxies. It contains a substantial young Mira population not seen elsewhere. This is interesting since the Magellanic Clouds, galaxies of the same morphology, are certainly forming stars today. However, we note that the comparison is not as informative as one would like. Glass et al. (1995), in their study of Miras in the Galactic bulge, indeed note that the period distribution from a given survey is highly dependent on the wavelength used. They found that variables that are IRAS sources have long periods, while blue photographic surveys are more sensitive to short-period Miras. This would explain the lack of long period Miras in the Magellanic Clouds. In addition, the OGLE survey was performed with optical filters, whereas Leo I and Fornax were observed in the NIR.

NGC 6822 is unique among the dwarf irregular galaxies of the Local Group. Its stellar spheroid and its HI disk rotate at right angles to each other (Demers et al. 2006a). NGC 6822 can therefore be considered to be a polar ring galaxy (PRG). Investigators of PRGs have found that almost all of these systems exhibit evidence of recent formation, possibly within the past 2 Gyr (Whitmore et al. 1990; Iodice et al. 2003). If this is also the case of NGC 6822, we need to identify the interaction that may have triggered a burst of star formation. This may then be seen today as a significant number of young Miras. 


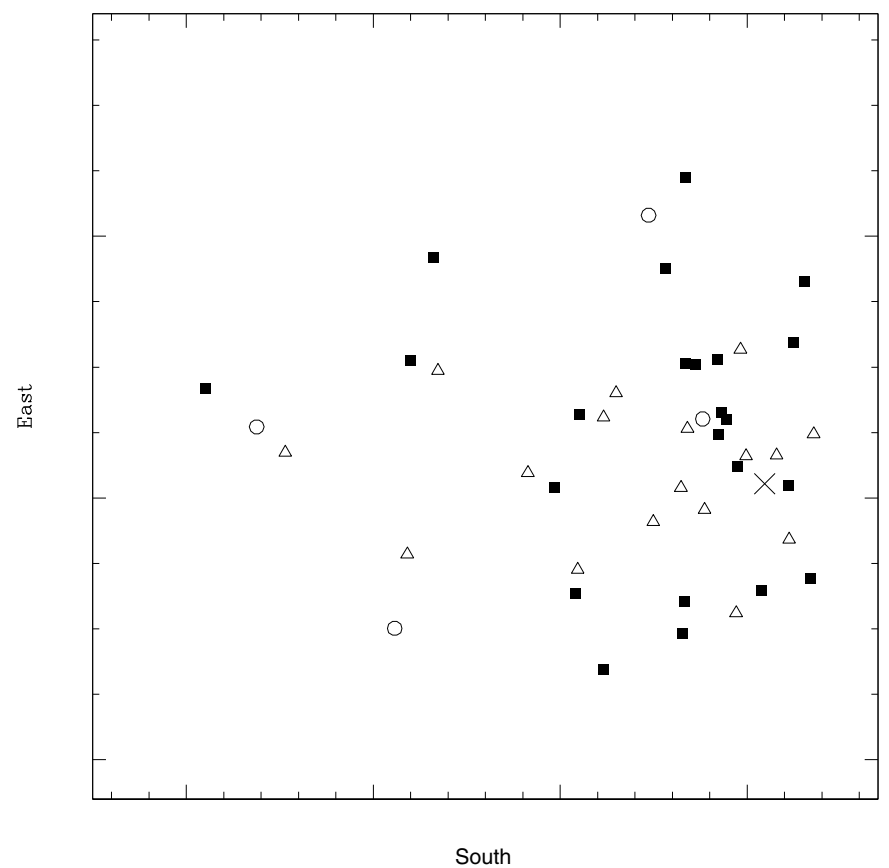

Fig. 9. Spatial distribution of the periodic and semi-periodic variables. Members of the older population are represented by circles, the intermediate-age by solid squares, and the younger ones by open triangles. The field of view corresponds to $4.4 \times 4.4 \mathrm{kpc}$, at the distance of NGC 6822 . The $\times$ marks the center of NGC 6822 .

The spatial distribution of the three age groups, shown in Fig. 9, is well mixed. Young and old stars are seen out to $\sim 3 \mathrm{kpc}$ from the center, where star formation is currently taking place. This implies that star formation has not been restricted exclusively to the center of the galaxy. We are thus dealing with a galaxy-wide global event.

Acknowledgements. This research is funded in parts (S.D.) by the Natural Sciences and Engineering Research Council of Canada. This publication makes use of data products from the Two Micron All Sky Survey, which is a joint project of the University of Massachusetts and the Infrared Processing and Analysis Center/California Institute of Technology, funded by the National Aeronautics and Space Administration and the National Science Foundation.

\section{References}

Antonello, E., Fugazza, D., Mantegazza, L., \& Stefanon, M. 2002, IBVS, 5254 Artigau, E., Doyon, R, Vallée, P., Riopel, M., \& Nadeau, D. 2004, in Groundbased Instrumentation for Astronomy, ed. A. F. M. Moorwood, \& I. Masanori, Proc. SPIE, 5492, 1479

Barnard, E. E. 1884, AN, 110, 125

Battinelli, P., \& Demers, S. 2011, in Why Galaxies care about AGB stars II, ed

F. Kerschbaum, T. Lebzelter, \& B. Wing. ASP Conf. Ser., to be published

Battinelli, P., Demers, S., \& Kunkel, W. E. 2006, A\&A, 451, 99

Battinelli, P., Demers, S., \& Mannucci, F. 2007, A\&A, 474, 35

Cioni, M.-R. L., \& Habing, H. J. 2005, A\&A, 429, 837

Clementini, G., Held, E. V., Baldacci, L., \& Rizzi, L. 2003, ApJ, 588, L85

Cook, K. H., Aaronson, M., \& Norris, J. 1986, ApJ, 305, 634

de Blok, W., \& Walter, F. 2000, ApJ, 537, L95

Demers, S., Battinelli, P., \& Kunkel, W. E. 2006a, ApJ, 636, L85

Demers, S., Battinelli, P., \& Artigau, É. 2006b, A\&A, 456, 905

Feast, M. W., Shitelock, P. A., \& Menzies, J. W. 2006, MNRAS, 21, 271

Gallart, C., Aparicio, A., Bertelli, G., \& Chiosi, C. 1996, AJ, 112, 1950

Glass, I. S., Whitelock, P. A., Catchpole, R. W., \& Feast, M. W. 1995, MNRAS, 273,383

Groenewegen, M. A. T. 2004, A\&A, 425, 595

Groenewegen, M. A. T., \& Blommaert, J. A. D. L. 2006, Mem. S. A. It., 77, 81

Habing, H. J., \& Whitelock, P. A. 2004, in Asymptotic Giant Branch Stars, ed. H. J. Habing, \& H. Olofsson (Springer), Chap. 8

Hodge, P. W. 1977, ApJS, 33, 69

Hodge, P. W. 1980, ApJ, 241, 125

Hodge, P., Smith, T., Eskridge, P., MacGillivray, H., \& Beard, S. 1991, ApJ, 379, 621

Hubble, E. P. 1925, ApJ, 62, 409

Iben, I., \& Renzini, A. 1983, ARA\&A, 21, 271

Iodice, E., Arnaboldi, M., Bournaud, F., et al. 2003, ApJ, 585, 730

Ita, Y., Tanabé, T., Matsunaga, N., et al. 2004, MNRAS, 347, 720

Kang, A., Sohn, Y.-J., Kim, H.-I., et al. 2006, A\&A, 454, 717

Kayser, S. E. 1967, AJ, 72, 134

Letarte, B., Demers, S., Battinelli, P., \& Kunkel, W. E. 2002, AJ, 123, 832

Lorenz, D., Nowotny, W., Lebzelter, T., et al. 2011, in Why Galaxies care about AGB stars II, ed. F. Kerschbaum, T. Lebzelter, \& B. Wing. ASP Conf. Ser., to be published

Mennickent, R. E., Gieren, W., Soszyński, I., \& Pietrzyński, G. 2006, A\&A, 450, 873

Menzies, J. W., Whitelock, P. A., Feast, M. W., \& Matshnaga, N. 2010, MNRAS, 406, 86

Pietrzyński, G., Gieren, W., Udalski, A., et al. 2004, AJ, 128, 2815

Schlegel, D., Finkbeiner, D., \& Davis, M. 1998, ApJ, 500, 525

Stellingwerf, R. F. 1978, ApJ, 224, 953

Stetson, P. B. 1994, PASP, 106, 250

Welch, D. L., \& Stetson, P. B. 1993, AJ, 105, 1813

Whitelock, P. A., Feast, M. W., \& van Leeuwen, F. 2008, MNRAS, 386, 313

Whitelock, P. A., Menzies, J. W., Feast, M. W., et al. 2009, MNRAS, 394, 795

Whitmore, B. C., Lucas, R. A., McElroy, D. B., et al. 1990, AJ, 100, 1489 